\title{
Non-supersymmetric deformations of the dual of a confining gauge theory
}

\author{
Vadim Borokhov \\ Lauritsen Laboratory of Physics \\ 452-48 Caltech, Pasadena, CA 91125, USA \\ E-mail: borokhov@its.caltech.edu

\section{Steven S. Gubser} \\ Joseph Henry Laboratories, Princeton University \\ Princeton, NJ 08544, USA \\ E-mail: ssgubser@princeton.edu
}

ABSTRACT: We introduce a computational technique for studying non-supersymmetric deformations of domain wall solutions of interest in AdS/CFT. We focus on the KlebanovStrassler solution, which is dual to a confining gauge theory. From an analysis of asymptotics we find that there are three non-supersymmetric deformations that leave the tendimensional supergravity solution regular and preserve the global bosonic symmetries of the supersymmetric solution. Also, we show that there are no regular near-extremal deformations preserving the global symmetries, as one might expect from the existence of a gap in the gauge theory.

Keywords: 1/N Expansion, Confinement, AdS-CFT and dS-CFT Correspondence. 


\section{Contents}

1. Introduction 1

2. The method 2

3. Metric ansatz and reduced action 1

4. Energy density of the perturbed solution 5

5. Formal solution 6

6. Asymptotic solutions 8

7. Non-extremal deformation 13

\section{Introduction}

One of the goals of AdS/CFT [迆]-[3] (see [沟 for a review) is the study of confinement. There are some examples in the literature (for example [5]-[7]) where confining gauge theories are dual to wholly non-singular geometries in supergravity. We consider it likely that many other confining theories exist whose duals are singular in supergravity: examples might include [8, 9]. In the absence of a better technical understanding of string theory in Ramond-Ramond backgrounds, it seems difficult to decide which singularities are physical (but see proposals in [10, 11]).

The purpose of this note is to explore non-singular, non-supersymmetric deformations of the non-singular supergravity dual of a confining gauge theory found in [6]. Briefly, the gauge theory is an $\mathcal{N}=1$ supersymmetric four-dimensional theory with gauge group $\mathrm{SU}(N) \times \mathrm{SU}(N+M)$, plus bifundamental matter that can be summarized by a quiver diagram. It is thought that the theory goes through a series of Seiberg dualities that eventually reduce the gauge group to $\mathrm{SU}(M)$, provided $M$ divides $N$. When the gauge group is $\mathrm{SU}(M)$, the bifundamental matter has disappeared, and the remaining theory of pure $\mathcal{N}=1$ supersymmetric glue confines.

The supergravity dual arises from D5-branes wrapped around a shrinking $S^{2}$, together with NS-NS three-form field strength and a D3-brane charge that grows logarithmically with radius. The geometry is not asymptotically anti-de Sitter; rather, it can be thought of as nearly $A d S_{5} \times T^{11}$ with a volume for $T^{11}$ that also grows logarithmically with radius. Metrically, it is a warped product of $\mathbb{R}^{3,1}$ with the deformed conifold:

$$
d s_{10}^{2}=\frac{1}{\sqrt{h}}\left(-d t^{2}+d \vec{x}^{2}\right)+\sqrt{h} d s_{6}^{2},
$$


where $h$ varies only in the conifold directions, whose Calabi-Yau metric is $d s_{6}^{2}$. In fact, global symmetries fix a radial direction in the deformed conifold, and $h$ varies only in this direction.

The solution (1.1) is supersymmetric 12, 13, and various properties appropriate to its interpretation as the dual of a confining gauge theory have been demonstrated in [6. As mentioned previously, our aim is to consider non-singular, non-supersymmetric deformations of it. Such solutions are bound to have meaning on the gauge theory side, as contrasted to singular deformations, which might or might not. A similar analysis has been carried out [14] for the related solution [7], where only Neveu-Schwarz three-form flux is present. Non-supersymmetric generalizations of [7] were also considered in [15], where it was found that a discrete series of disconnected non-supersymmetric vacua exist. A interpretation on the gauge theory side was suggested in [16].

The plan of the paper is as follows. We introduce our calculation method in section 2 we summarize the unperturbed solution in section 3 , we obtain a general form of the renormalized mass density of the perturbed solution in section 난 and we consider formal and asymptotic properties of Lorentz-invariant perturbed solutions in sections 5 and 6 . At the end of section 6 we reach the conclusion that there are three regular non-supersymmetric deformations which preserve the global symmetries of the unperturbed, supersymmetric solution. In section 0 we study perturbations that break boost symmetries in four dimensions but retain all other symmetries, with the eventual conclusion that there are no near-extremal perturbations with regular horizon. This squares nicely with the expectation that the field theory has a gap.

\section{The method}

A generally useful trick for finding supersymmetric solutions is to parametrize the shape of the compact dimensions by scalars, and then to show that preserved supersymmetry demands that these scalars obey the gradient flow equations of some function $W$. This method goes by the name of "attractor equations" in the study of supersymmetric black holes in four dimensions [17], and it has also been used extensively in AdS/CFT, with $W$ having the interpretation of a superpotential - see for example [18, and [19] for some remarks on the similarity of the attractor equations and the superpotential methods for finding domain wall solutions. "Superpotential methods" are not in fact restricted to supersymmetric situations [20, 21]: $W$ can be related to Hamilton's principle function [22, and only particular forms (specified by particular choices of integration constants) correspond to supersymmetric situations.

Our calculational technique for studying perturbations relies on starting with a solution generated from a known superpotential. Abstractly, the problem may be cast in the form of supersymmetric quantum mechanics, with radial variable $u$ playing the role of time. The radial lagrangian is

$$
\begin{aligned}
L & =-\frac{1}{2} G_{a b} \frac{d \phi^{a}}{d u} \frac{d \phi^{b}}{d u}-V(\phi) \\
& =-\frac{1}{2} G_{a b}\left(\frac{d \phi^{a}}{d u}-G^{a c} \frac{\partial W}{\partial \phi^{c}}\right)\left(\frac{d \phi^{b}}{d u}-G^{b d} \frac{\partial W}{\partial \phi^{d}}\right)+\frac{1}{2} \frac{d W}{d u},
\end{aligned}
$$


where

$$
V=\frac{1}{8} G^{a b} \frac{\partial W}{\partial \phi^{a}} \frac{\partial W}{\partial \phi^{b}} .
$$

(The scalars $\phi^{a}$ here include components of the metric which participate in the solution). The gradient flow equations are

$$
\frac{d \phi^{a}}{d u}=\frac{1}{2} G^{a b} \frac{\partial W}{\partial \phi^{b}},
$$

and since the problem is gravitational, there is also a "zero-energy" constraint that comes from the $G_{u u}$ Einstein equation:

$$
-\frac{1}{2} G_{a b} \frac{d \phi^{a}}{d u} \frac{d \phi^{b}}{d u}+V(\phi)=0 .
$$

Evidently, a solution to (2.3) will also solve the equations of motion for (2.1) as well as constraint equation (2.4). All this is a many-times-told story. Where we are introducing something novel is to continue to use the superpotential as much as possible to study small perturbations to a solution of (2.3) that in general satisfy the second order equations of motion, but not (2.3) itself. Let us expand around a given solution, $\phi_{0}^{a}$, to (2.3):

$$
\phi^{a}=\phi_{0}^{a}+\alpha \bar{\phi}^{a}+O\left(\alpha^{2}\right)
$$

with a small positive constant $\alpha$. It is convenient to introduce further functions

$$
\xi_{a}=G_{a b}\left(\phi_{0}\right)\left(\frac{d \bar{\phi}^{b}}{d u}-N_{d}^{b}\left(\phi_{0}\right) \bar{\phi}^{d}\right) \quad \text { where } \quad N_{a}^{b}=\frac{1}{2} \frac{\partial}{\partial \phi^{a}}\left(G^{b c} \frac{\partial W}{\partial \phi^{c}}\right) .
$$

Then the linearized equations of motion can be represented as

$$
\begin{aligned}
\frac{d \xi_{a}}{d u}+\xi_{b} N^{b}{ }_{a}\left(\phi_{0}\right) & =0, \\
\frac{d \bar{\phi}^{a}}{d u}-N^{a}{ }_{b}\left(\phi_{0}\right) \bar{\phi}^{b} & =G^{a b}\left(\phi_{0}\right) \xi_{b} .
\end{aligned}
$$

The constraint can be rephrased as $\xi_{a} d \phi_{0}^{a} / d u=0$.

The set of equations (2.8) is a trivial consequence of the definition of $\xi_{a}$. Equations (2.7) may be demonstrated by plugging the expansion (2.5) into the equations of motion, in the form

$$
\begin{aligned}
\frac{d}{d u}\left(G_{a b}\left(\phi^{\prime b}-\frac{1}{2} G^{b c} \partial_{c} W\right)\right)+\frac{1}{2} & \left(\partial_{a} \partial_{b} W-\left(\partial_{a} G_{b c}\right) G^{c d} \partial_{d} W\right)\left(\phi^{\prime b}-\frac{1}{2} G^{b k} \partial_{k} W\right)- \\
& -\frac{1}{2}\left(\partial_{a} G_{b c}\right)\left(\phi^{\prime b}-\frac{1}{2} G^{b d} \partial_{d} W\right)\left(\phi^{\prime c}-\frac{1}{2} G^{c k} \partial_{k} W\right)=(\mathbb{Q} 9)
\end{aligned}
$$

where primes mean $d / d u$. The constraint can be written as

$$
G_{a b}\left(\phi^{\prime a}-\frac{1}{2} G^{a c} \partial_{c} W\right)\left(\phi^{\prime b}+\frac{1}{2} G^{b d} \partial_{d} W\right)=0
$$

from which $\xi_{a} d \phi_{0}^{a} / d u=0$ easily follows. 
Roughly speaking, $\xi_{a}$ and $\bar{\phi}^{a}$ are canonically conjugate. It is easy to see that $\xi_{a}$ describe deformations of the gradient flow equations. Namely, if all $\xi_{a}$ vanish then the deformation is supersymmetric.

Let $\{X\}$ be integration constants parameterizing linearly independent solutions to (2.7) subject to the constraint and a set of constants $\{Z\}$ parameterize linearly independent solutions to (2.8) with vanishing right-hand side. Since (2.8) is a set of nonhomogeneous linear differential equations, general solution for $\bar{\phi}$ is a sum of solutions parametrized by $\{X\}$ and solutions parameterized by $\{Z\}$. Clearly, $\{X\}$ describe the non-supersymmetric deformations, whereas $\{Z\}$ correspond to supersymmetric ones. For example, deformation generated by $u \rightarrow u+\alpha$ is supersymmetric: $\xi_{a}=0, \bar{\phi}^{a}=\phi_{0}^{\prime a}$. As we shall see in section 6 , only specific superpositions of supersymmetric and non-supersymmetric deformations may prove to be regular.

Using (2.7) and (2.8) is convenient as a calculational scheme because one can solve first for the $\xi_{a}$ through first order equations, then for $\bar{\phi}^{a}$ through more first order equations, rather than tackling the second order equations directly. Let us now see the method in action for a non-trivial example.

\section{Metric ansatz and reduced action}

The ansatz of [6] (see also [23]) is the most general one consistent with the global symmetries of the field theory dual, namely $\mathrm{SU}(2) \times \mathrm{SU}(2)$ of flavor and $\mathrm{U}(1)_{R}$. The metric is

$$
\begin{aligned}
d s_{10}^{2}= & e^{2 p-x+2 A}\left(-d t^{2}+d x^{i} d x^{i}\right)+e^{2 p-x+8 A} d u^{2}+ \\
& +\left[e^{-6 p-x} g_{5}^{2}+e^{x+y}\left(g_{1}^{2}+g_{2}^{2}\right)+e^{x-y}\left(g_{3}^{2}+g_{4}^{2}\right)\right]
\end{aligned}
$$

and the forms are

$$
\begin{aligned}
H_{3} & \left.=d u \wedge\left[f^{\prime}(u) g_{1} \wedge g_{2}+k^{\prime}(u) g_{3} \wedge g_{4}\right)\right]+\frac{1}{2}[k(u)-f(u)] g_{5} \wedge\left(g_{1} \wedge g_{3}+g_{2} \wedge g_{4}\right) \\
F_{3} & =F(u) g_{1} \wedge g_{2} \wedge g_{5}+[2 P-F(u)] g_{3} \wedge g_{4} \wedge g_{5}+F^{\prime}(u) d u \wedge\left(g_{1} \wedge g_{3}+g_{2} \wedge g_{4}\right) \\
F_{5} & =\mathcal{F}_{5}+\mathcal{F}_{5}^{*}, \quad \mathcal{F}_{5}=K(u) g_{1} \wedge g_{2} \wedge g_{3} \wedge g_{4} \wedge g_{5} \\
K(u) & =k(u) F(u)+f(u)[2 P-F(u)],
\end{aligned}
$$

where $P$ is a constant. Explicit expressions for the one-forms $g_{i}$ can be found in [6].

The eight scalars that will participate in the radial lagrangian are $\phi^{a}=(x, y, p, A, f$, $k, F, \Phi)$. To obtain the radial lagrangian, one may start with the type IIB supergravity action (in the by-now-standard form where $F_{5}^{2}$ appears but is set to zero by imposing selfduality after obtaining the equations of motion) and perform the integrals over the angular directions as well as factoring out the volume of four-dimensional Minkowski spacetime. The result is the reduced action: up to an overall factor,

$$
S\left[\phi^{a}\right]=-\frac{2 \mathrm{Vol}_{4}}{\kappa_{5}^{2}} \int d u\left(-\frac{1}{2} G_{a b} \phi^{\prime a} \phi^{\prime b}-V(\phi)\right)
$$


where

$$
\begin{aligned}
G_{a b} \phi^{\prime a} \phi^{\prime b}= & -6 A^{\prime 2}+x^{\prime 2}+\frac{1}{2} y^{\prime 2}+6 p^{\prime 2}+ \\
& +\frac{1}{4}\left(\Phi^{\prime 2}+e^{-\Phi-2 x}\left(e^{-2 y} f^{\prime 2}+e^{2 y} k^{\prime 2}\right)+2 e^{\Phi-2 x} F^{\prime 2}\right),
\end{aligned}
$$

and

$$
\begin{aligned}
V(\phi)= & \frac{1}{4} e^{8 A-4 p-4 x}-e^{8 A+2 p-2 x} \cosh y+\frac{1}{4} e^{8 p+8 A} \sinh ^{2} y+ \\
& +\frac{1}{8} e^{8 p+8 A}\left(\frac{1}{2} e^{-\Phi-2 x}(f-k)^{2}+e^{\Phi-2 x}\left(e^{-2 y} F^{2}+e^{2 y}(2 P-F)^{2}\right)+e^{-4 x} K^{2}\right) .
\end{aligned}
$$

The superpotential is (cf. [23)

$$
W(\phi)=e^{4 A+4 p} \cosh y+e^{4 A-2 p-2 x}+\frac{1}{2} e^{4 A+4 p-2 x} K .
$$

To write down the supersymmetric solution of [6] , it is convenient to change radial variables by $d u=-e^{-4 A-4 p} d \tau$. Then

$$
d s_{10}^{2}=\frac{1}{\sqrt{h(\tau)}}\left(-d t^{2}+d x^{i} d x^{i}\right)+\sqrt{h(\tau)} d s_{6}^{2},
$$

where $d s_{6}^{2}$ is metric of the deformed conifold,

$$
\begin{aligned}
d s_{6}^{2} & =\frac{1}{2} \epsilon^{4 / 3} N(\tau)\left[\frac{1}{3 N^{3}(\tau)}\left(d \tau^{2}+g_{5}^{2}\right)+\cosh ^{2} \frac{\tau}{2}\left(g_{3}^{2}+g_{4}^{2}\right)+\sinh ^{2} \frac{\tau}{2}\left(g_{1}^{2}+g_{2}^{2}\right)\right], \\
N(\tau) & =\frac{(\sinh 2 \tau-2 \tau)^{1 / 3}}{2^{1 / 3} \sinh \tau}, \quad \epsilon=12^{1 / 4}, \\
h(\tau) & =a \frac{2^{2 / 3}}{4} \int_{\tau}^{\infty} d x \frac{x \operatorname{coth} x-1}{\sinh ^{2} x}(\sinh (2 x)-2 x)^{1 / 3}, \quad a=\frac{64 P^{2}}{\epsilon^{8 / 3}}, \\
f_{0}(\tau) & =-\frac{P(\tau \operatorname{coth} \tau-1)(\cosh \tau-1)}{\sinh \tau}, \quad k_{0}(\tau)=-\frac{P(\tau \operatorname{coth} \tau-1)(\cosh \tau+1)}{\sinh \tau}, \\
F_{0}(\tau) & =\frac{P(\sinh \tau-\tau)}{\sinh \tau}, \quad \Phi_{0}=0 .
\end{aligned}
$$

\section{Energy density of the perturbed solution}

Let the surface $\partial \mathcal{M}_{u} \simeq R^{3,1} \times T^{11}$ at fixed $u$ be the boundary of the interior region $\mathcal{M}_{u}$. Consider the boundary metric $\gamma_{\mu \nu}$, where

$$
d s^{2}=g_{u u} d u^{2}+\gamma_{\mu \nu} \omega^{\mu} \omega^{\nu}, \quad \omega^{\mu}=\left(d t, \omega^{\alpha}\right), \quad \omega^{\alpha}=\left(d x^{i}, g_{1}, \ldots, g_{5}\right) .
$$

Following [24] we define the quasilocal stress-energy tensor

$$
T_{\mu \nu}=\frac{1}{8 \pi G}\left(\Theta_{\mu \nu}-\Theta \gamma_{\mu \nu}+\frac{2}{\sqrt{|\gamma|}} \frac{\delta S_{c t}}{\delta \gamma^{\mu \nu}}\right),
$$


where $\Theta_{\mu \nu}$ is the extrinsic curvature

$$
\Theta_{\mu \nu}=\frac{1}{2 \sqrt{g_{u u}}} \partial_{u} \gamma_{\mu \nu}, \quad \Theta=\Theta_{\mu \nu} \gamma^{\mu \nu},
$$

and a counterterm action $S_{c t}$ must be chosen to cancel divergences that appear when $u$ approaches its minimum value ( $\tau$ goes to infinity). For a supersymmetric solution the counterterm is associated with tension of the boundary $\partial \mathcal{M}_{u}$ :

$$
S_{c t}=\int_{\partial \mathcal{M}_{u}} d t \wedge d x^{i} \wedge g_{1} \wedge \cdots \wedge g_{5} W(\phi)
$$

so that

$$
\frac{\delta S_{c t}}{\delta \gamma^{\mu \nu}}=\frac{1}{2} W \gamma_{\mu \nu}
$$

Let a spacelike surface $\Sigma$ with a metric $\sigma_{\alpha \beta}$ be normal to the timelike unit vector $\frac{1}{N_{\Sigma}} \frac{\partial}{\partial t}$ : that is,

$$
\gamma_{\mu \nu} \omega^{\mu} \omega^{\nu}=-N_{\Sigma}^{2} d t^{2}+\sigma_{\alpha \beta} \omega^{\alpha} \omega^{\beta} .
$$

Then the mass density $M$ is given by

$$
M=\int_{\Sigma} g_{1} \wedge \cdots \wedge g_{5} \mu, \quad \mu=\sqrt{|\sigma|} \frac{1}{N_{\Sigma}} T_{t t} .
$$

It is possible that this does not vanish even when the perturbed solution is Lorentz invariant. In such a case, the mass density $M$ is interpreted as a contribution to the four-dimensional cosmological constant. (But it is consistent that the four-dimensional geometry is Minkowski space, because the four-dimensional Newton coupling vanishes for these non-compact geometries). If the solution is not Lorentz invariant (as for a nonextremal deformation), then one should also obtain the spatial components of $T_{\mu \nu}$. For supersymmetric solution we have

$$
T_{t t}=\frac{3}{8 \pi G} e^{-x / 2+p-2 A}\left(A^{\prime}-\frac{1}{3} W\right), \quad \mu=\frac{3}{8 \pi G}\left(A^{\prime}-\frac{1}{3} W\right),
$$

which are proportional to the gradient flow equation for $A$. Therefore energy and mass densities of any supersymmetric solution vanish.

For a non-supersymmetric solution additional counterterms might be needed [25, 26. If counterterms do not break supersymmetry, their contribution to the energy density has the form $O\left[d \phi^{a} / d u-1 / 2 G^{a b} \partial W / \partial \phi^{b}\right]$. Therefore, for a perturbed solution (2.5), we have $T_{t t}$ and $\mu$ of the form $\alpha O[\xi]+O\left(\alpha^{2}\right)$.

\section{Formal solution}

The linearized equations of motion (2.7) and (2.8) admit formal solutions in terms of path ordered exponentials, but this is not very useful in practice unless the "connection" matrix $N_{b}^{a}\left(\phi_{0}\right)$ is diagonal or triangular - that is, unless the equations decouple, or can be solved iteratively. This occurs only partially for our case, as we shall develop in this section by examining the explicit form of the equations and extracting the simplest combinations of them that we can. 
First, let us use the radial variable $\tau \mathrm{m}\left(d u=-e^{-4 A_{0}-4 p_{0}} d \tau\right)$, so that (2.7) and (2.8) become

$$
\begin{aligned}
\dot{\xi}_{a}+\xi_{b} M^{b}{ }_{a}\left(\phi_{0}\right) & =0 \\
\dot{\bar{\phi}}^{a}-M^{a}{ }_{b}\left(\phi_{0}\right) \bar{\phi}^{b} & =-e^{-4 A_{0}-4 p_{0}} G^{a b}\left(\phi_{0}\right) \xi_{b}, \\
M^{b}{ }_{a} & =-e^{-4 A_{0}-4 p_{0}} N^{b}{ }_{a} .
\end{aligned}
$$

Given a vector, like $\xi_{a}$, we will have frequent occasion to use the shorthand notation $\xi_{3+4}=\xi_{3}+\xi_{4}$, or $\xi_{5-6}=\xi_{5}-\xi_{6}$.

First let us deal with the first line of (5.1). The vector

$$
V=\left(V^{a}\right)=\left(3,0,-1,1, \frac{3}{P}[2 f P+F(k-f)], \frac{3}{P}[2 f P+F(k-f)], 0,0\right)
$$

is annihilated by $M_{b}^{a}$ : that is, $M_{b}^{a} V^{b}=0$. It follows that

$$
\dot{\eta}+\frac{3}{P}\left[2 f_{0} P+F_{0}\left(k_{0}-f_{0}\right)\right] \dot{\xi}_{5+6}=0,
$$

where $\eta=3 \xi_{1}-\xi_{3}+\xi_{4}$ and $\xi_{5+6}=\xi_{5}+\xi_{6}$ as per our shorthand notation explained above. We also find

$$
\dot{\xi}_{5+6}+\frac{1}{3} e^{-2 x_{0}} P \eta=0,
$$

and now we can solve

$$
\begin{aligned}
\eta(\tau) & =X_{\eta} \exp \left(\int_{\tau_{0}}^{\tau} d \tau e^{-2 x_{0}}\left[2 f_{0} P+F_{0}\left(k_{0}-f_{0}\right)\right]\right) \\
\xi_{5+6}(\tau) & =X_{5+6}-\frac{P}{3} \int_{\tau_{0}}^{\tau} d \tau e^{-2 x_{0}} \eta(\tau),
\end{aligned}
$$

where $X_{\eta}$ and $X_{5+6}$ are integration constants. It can be further shown that

$$
\begin{aligned}
\dot{\xi}_{5-6}+\xi_{7} & =\frac{1}{3} e^{-2 x_{0}}\left(F_{0}-P\right) \eta \\
\dot{\xi}_{7}+\cosh \left(2 y_{0}\right) \xi_{5-6} & =\frac{1}{6} e^{-2 x_{0}}\left(f_{0}-k_{0}\right) \eta-\sinh \left(2 y_{0}\right) \xi_{5+6} .
\end{aligned}
$$

Once $\xi_{5-6}$ and $\xi_{7}$ are determined, we can find $\xi_{8}$ using

$$
\dot{\xi}_{8}=e^{2 y_{0}}\left(2 P-F_{0}\right) \xi_{5}+e^{-2 y_{0}} F_{0} \xi_{6}+\frac{1}{2}\left(f_{0}-k_{0}\right) \xi_{7} .
$$

Using the constraint $\xi_{a} \dot{\phi}_{0}^{a}=0$, together with the solution for $\eta$, we may solve for $\xi_{3}$ and $\xi_{4}$, and then we find two coupled linear differential equations for $\xi_{1}$ and $\xi_{2}$. This completes a formal solution of $\dot{\xi}_{a}+\xi_{b} M^{b}{ }_{a}=0$ together with $\xi_{a} \dot{\phi}_{0}^{a}=0$.

We have still to solve the second equation in (5.1), that is, $\dot{\bar{\phi}}^{a}-M^{a}{ }_{b}\left(\phi_{0}\right) \bar{\phi}^{b}=-e^{-4 A_{0}-4 p_{0}} \times$ $G^{a b}\left(\phi_{0}\right) \xi_{b}$. For $\bar{\Phi}$ we have

$$
\dot{\bar{\Phi}}=-4 e^{-4 A_{0}-4 p_{0}} \xi_{8} .
$$


Functions $Y(\tau)=\bar{x}(\tau)-3 \bar{A}(\tau), \bar{\phi}_{3+4}(\tau)=\bar{p}(\tau)+\bar{A}(\tau)$ and $\bar{y}$ satisfy

$$
\begin{aligned}
\dot{\bar{\phi}}_{3+4}+e^{-2 x_{0}-6 p_{0}} \bar{\phi}_{3+4}+e^{-2 x_{0}-6 p_{0}} Y & =\frac{1}{6} e^{-4 p_{0}-4 A_{0}}\left(\xi_{4}-\xi_{3}\right) \\
\dot{Y}+4 \cosh \left(y_{0}\right) \bar{\phi}_{3+4}+\sinh \left(y_{0}\right) \bar{y} & =-e^{-4 p_{0}-4 A_{0}}\left(\xi_{1}+\frac{1}{2} \xi_{4}\right) \\
\dot{\bar{y}}+\cosh \left(y_{0}\right) \bar{y}+4 \sinh \left(y_{0}\right) \bar{\phi}_{3+4} & =-2 e^{-4 p_{0}-4 A_{0}} \xi_{2} .
\end{aligned}
$$

For $\bar{\phi}_{5-6}=\bar{f}-\bar{k}$ and $\bar{F}$ one obtains

$$
\begin{aligned}
\dot{\bar{F}}-\frac{1}{2} \bar{\phi}_{5-6}= & \frac{1}{2}\left(f_{0}-k_{0}\right)\left(4 \bar{\phi}_{3+4}-\bar{\Phi}\right)-2 e^{2 x_{0}-4 p_{0}-4 A_{0}} \xi_{7}, \\
\dot{\bar{\phi}}_{5-6}-2 \cosh \left(2 y_{0}\right) \bar{F}= & 4\left(\sinh \left(2 y_{0}\right) F_{0}-e^{2 y_{0}} P\right) \bar{y}+2\left(\cosh \left(2 y_{0}\right) F_{0}-e^{2 y_{0}} P\right) \times \\
& \times\left(4 \bar{\phi}_{3+4}+\bar{\Phi}\right)+8 e^{2 x_{0}-4 p_{0}-4 A_{0}} \sinh \left(2 y_{0}\right)\left(\xi_{5}-\xi_{6}\right) .
\end{aligned}
$$

A function $\bar{\phi}_{5+6}=\bar{f}+\bar{k}$ satisfies

$$
\begin{aligned}
\dot{\bar{\phi}}_{5+6}= & -4\left(e^{2 y_{0}} P+\cosh \left(2 y_{0}\right) F_{0}\right) \bar{y}+2\left(\sinh \left(2 y_{0}\right) F_{0}-e^{2 y_{0}} P\right)\left(4 \bar{\phi}_{3+4}+\bar{\Phi}\right)+ \\
& +2 \sinh \left(2 y_{0}\right) \bar{F}-8 e^{2 x_{0}-4 p_{0}-4 A_{0}} \cosh \left(2 y_{0}\right)\left(\xi_{5}+\xi_{6}\right) .
\end{aligned}
$$

Finally, for $\bar{A}$ we have

$$
\begin{aligned}
\dot{\bar{A}}+ & e^{-2 x_{0}}\left(2 f_{0} P+F_{0}\left(k_{0}-f_{0}\right)\right) \bar{A}= \\
= & \frac{1}{3} \sinh \left(y_{0}\right) \bar{y}+\frac{2}{3}\left[2 e^{-2 x_{0}} f_{0} P+e^{-2 x_{0}} F_{0}\left(k_{0}-f_{0}\right)-e^{-2 x_{0}-6 p_{0}}+2 \cosh \left(y_{0}\right)\right] \bar{\phi}_{3+4}- \\
& -\frac{1}{3} e^{-2 x_{0}}\left[2 f_{0} P+F_{0}\left(k_{0}-f_{0}\right)+2 e^{-6 p_{0}}\right] Y+\frac{1}{6} e^{-2 x_{0}}\left(2 P-F_{0}\right) \bar{f}+\frac{1}{6} e^{-2 x_{0}} F_{0} \bar{k}+ \\
& +\frac{1}{6} e^{-2 x_{0}}\left(k_{0}-f_{0}\right) \bar{F}+\frac{1}{6} e^{-4 p_{0}-4 A_{0}} \xi_{4} .
\end{aligned}
$$

This completes a formal solution of the linearized equations (5.1).

\section{Asymptotic solutions}

Unfortunately, even the partially decoupled equations that we found in the previous section do not appear to admit analytic solutions. What we can do, however, is to give a complete treatment of the asymptotics in the regions $\tau \rightarrow 0$ (in the interior, where we may require that the solution be completely regular) and at $\tau \rightarrow \infty$, where we can sensibly require that the asymptotics is unchanged at the leading order from the supersymmetric solution and that the energy density is finite.

We will use the algorithm described in the previous section to find asymptotic form of the perturbations $\bar{\phi}$ in the regions $\tau \rightarrow 0$ and $\tau \rightarrow \infty$.

Large $\tau$ : observing that

$$
\begin{aligned}
& \left(\frac{\partial}{\partial \phi} W\right)\left(\phi_{0}\right) \sim \frac{e^{4 / 3 \tau}}{\tau} \quad \text { for } \quad \phi=(x, p, f, k), \quad\left(\frac{\partial}{\partial A} W\right)\left(\phi_{0}\right) \sim e^{4 / 3 \tau}, \\
& \left(\frac{\partial}{\partial \phi} W\right)\left(\phi_{0}\right) \sim e^{\tau / 3} \quad \text { for } \quad \phi=(y, F), \quad G^{a a}\left(\phi_{0}\right) e^{-4 p_{0}-4 A_{0}} \sim \tau e^{-4 / 3 \tau},
\end{aligned}
$$


we conclude that we must solve for $\xi$ up to the terms $\sim 1 / \tau$ at infinity; find $\bar{x}, \bar{f}$, and $\bar{k}$ up to $\tau e^{-4 / 3 \tau} ; \bar{p}$ and $\bar{A}$ up to $e^{-4 / 3 \tau}$; and $\bar{y}$ and $\bar{F}$ up to $e^{-\tau / 3}$. The solution for $\xi(\tau)$ has the following form:

$$
\begin{aligned}
& \xi_{1}=X_{1}^{\infty} e^{2 \tau}-4 X_{1}^{\infty} \tau-4 X_{1}^{\infty}+6 P X_{5-6}^{\infty}-P X_{5+6}^{\infty}+2 X_{2}^{\infty}+O\left(e^{-\tau}\right), \\
& \xi_{2}=2\left(P X_{5-6}^{\infty}-X_{1}^{\infty}\right) e^{\tau} \tau+X_{2}^{\infty} e^{\tau}+O\left(e^{-\tau}\right), \quad \xi_{3}=3 X_{1}^{\infty} e^{2 \tau}-12 X_{1}^{\infty} \tau+O\left(e^{-\tau}\right), \\
& \xi_{4}=-18 P X_{5-6}^{\infty}+3 P X_{5+6}^{\infty}-6 X_{2}^{\infty}+12 X_{1}^{\infty}+O\left(e^{-\tau}\right), \\
& \xi_{5}=\frac{1}{2} X_{5-6}^{\infty} e^{\tau}+\frac{1}{2} X_{5+6}^{\infty}+O\left(e^{-\tau}\right), \quad \xi_{6}=-\frac{1}{2} X_{5-6}^{\infty} e^{\tau}+\frac{1}{2} X_{5+6}^{\infty}+O\left(e^{-\tau}\right), \\
& \xi_{7}=-X_{5-6}^{\infty} e^{\tau}+O\left(e^{-\tau}\right), \quad \xi_{8}=\left(X_{5+6}^{\infty}-2 X_{5-6}^{\infty}\right) P \tau+X_{8}^{\infty}+O\left(e^{-\tau}\right) .
\end{aligned}
$$

where $X_{1}^{\infty}, X_{2}^{\infty}, X_{5-6}^{\infty}, X_{5+6}^{\infty}, X_{8}^{\infty}$ are integration constants. The other integration constants parametrize solutions which are $O\left(e^{-\tau}\right)$. For the dilaton deformation we have

$$
\begin{aligned}
\bar{\Phi}= & Z_{8}^{\infty}+3^{1 / 3} 4 P X_{5+6}^{\infty} e^{-4 / 3 \tau}(3+4 \tau)-3^{1 / 3} 8 P X_{5-6}^{\infty} e^{-4 / 3 \tau}(3+4 \tau)+ \\
& +3^{1 / 3} 16 X_{8}^{\infty} e^{-4 / 3 \tau}+\cdots,
\end{aligned}
$$

where $Z_{8}^{\infty}$ is an integration constant. To keep $\Phi(\infty)$ fixed, we set

$$
Z_{8}^{\infty}=0
$$

For $\bar{f}$ and $\bar{k}$ we have

$$
\begin{aligned}
\bar{f}= & \frac{1}{2} Z_{5+6}^{\infty}+\frac{1}{2} Z_{7}^{\infty} e^{-\tau}-\frac{1}{2} P Z_{2}^{\infty} e^{-\tau}(1+2 \tau)+3^{1 / 3} 12 P X_{2}^{\infty}\left(e^{-\tau / 3}-4 \tau e^{-4 / 3 \tau}\right)+ \\
& +3^{1 / 3} P^{2} X_{5-6}^{\infty}\left(-96 \tau^{2} e^{-4 / 3 \tau}-96 \tau e^{-4 / 3 \tau}-90 e^{-\tau / 3}\right)+3^{1 / 3} 24 P^{2} X_{5+6}^{\infty} \tau e^{-4 / 3 \tau}+ \\
& +P Z_{Y}^{\infty}\left(e^{4 / 3 \tau}+O\left(\tau e^{\tau / 3}\right)\right)+Z_{5-6}^{\infty}\left(\frac{1}{2} e^{\tau}-2 \tau\right)+3^{1 / 3} 6 P X_{1}^{\infty} e^{2 / 3 \tau}+\cdots, \\
\bar{k}= & \frac{1}{2} Z_{5+6}^{\infty}-\frac{1}{2} Z_{7}^{\infty} e^{-\tau}+\frac{1}{2} P Z_{2}^{\infty} e^{-\tau}(1+2 \tau)-3^{1 / 3} 12 P X_{2}^{\infty}\left(e^{-\tau / 3}+4 \tau e^{-4 / 3 \tau}\right)+ \\
& +3^{1 / 3} P^{2} X_{5-6}^{\infty}\left(-96 \tau^{2} e^{-4 / 3 \tau}-96 \tau e^{-4 / 3 \tau}+90 e^{-\tau / 3}\right)+3^{1 / 3} 24 P^{2} X_{5+6}^{\infty} \tau e^{-4 / 3 \tau}+ \\
& +P Z_{Y}^{\infty}\left(e^{4 / 3 \tau}+O\left(\tau e^{\tau / 3}\right)\right)+Z_{5-6}^{\infty}\left(-\frac{1}{2} e^{\tau}-2 \tau\right)+3^{1 / 3} 6 P X_{1}^{\infty} e^{2 / 3 \tau}+\cdots,
\end{aligned}
$$

where $Z_{5-6}^{\infty}, Z_{5+6}^{\infty}, Z_{Y}^{\infty}, Z_{7}^{\infty}$, and $Z_{2}^{\infty}$ are integration constants. Although $k_{0}(\tau)$ and $f_{0}(\tau)$ are divergent at infinity, the divergence is linear in $\tau$. Therefore, we require vanishing of the exponentially divergent terms in (6.5):

$$
X_{1}^{\infty}=Z_{Y}^{\infty}=Z_{5-6}^{\infty}=0 .
$$

For the rest of deformations $\bar{\phi}$ we have

$$
\begin{aligned}
\bar{x}= & -3^{1 / 3} 48 P X_{5-6}^{\infty} \tau e^{-4 / 3 \tau}+Z_{4}^{\infty}\left(3 \tau^{-4 / 3} e^{4 / 3 \tau}+O\left(e^{-2 / 3 \tau}\right)\right)- \\
& -\frac{3^{1 / 3}}{4^{4 / 3} P} Z_{5+6}^{\infty} \tau^{-4 / 3} e^{4 / 3 \tau} \Gamma\left(\frac{4}{3}, \frac{4}{3} \tau\right)+\cdots, \\
\bar{y}= & 3^{1 / 3} 16 P X_{5-6}^{\infty} e^{-\tau / 3}(3-2 \tau)-3^{1 / 3} 16 X_{2}^{\infty} e^{-\tau / 3}+\cdots,
\end{aligned}
$$




$$
\begin{aligned}
\bar{p}= & -3^{1 / 3} P X_{5-6}^{\infty} e^{-4 / 3 \tau}(10+16 \tau)+Z_{4}^{\infty}\left(-\tau^{-4 / 3} e^{4 / 3 \tau}+O\left(e^{-2 / 3 \tau}\right)\right)+ \\
& +3^{1 / 3} 4 e^{-4 / 3 \tau}\left(-2 X_{2}^{\infty}+P X_{5+6}^{\infty}\right)+\frac{1}{3^{2 / 3} 4^{4 / 3} P} Z_{5+6}^{\infty} \tau^{-4 / 3} e^{4 / 3 \tau} \Gamma\left(\frac{4}{3}, \frac{4}{3} \tau\right)+\cdots \\
\bar{A}= & 3^{1 / 3} P X_{5-6}^{\infty} e^{-4 / 3 \tau}(67-8 \tau)+Z_{4}^{\infty}\left(\tau^{-4 / 3} e^{4 / 3 \tau}+O\left(e^{-2 / 3 \tau}\right)\right)- \\
& -\frac{1}{3^{2 / 3} 4^{4 / 3} P} Z_{5+6}^{\infty} \tau^{-4 / 3} e^{4 / 3 \tau} \Gamma\left(\frac{4}{3}, \frac{4}{3} \tau\right)-3^{1 / 3} 6 X_{2}^{\infty} e^{-4 / 3 \tau}-3^{4 / 3} P X_{5+6}^{\infty} e^{-4 / 3 \tau}+\cdots, \\
\bar{F}= & 3^{1 / 3} P^{2} X_{5-6}^{\infty} e^{-\tau / 3}(126-48 \tau)-3^{1 / 3} 36 P X_{2}^{\infty} e^{-\tau / 3}+\cdots,
\end{aligned}
$$

where $Z_{4}^{\infty}$ is a constant and $\Gamma(4 / 3, z)$ is the incomplete gamma function ${ }^{1}$

$$
\Gamma\left(\frac{4}{3}, z\right)=e^{-z}\left(z^{1 / 3}+\frac{1}{3} z^{-2 / 3}+O\left(\frac{1}{z^{5 / 3}}\right)\right), \quad z \rightarrow \infty .
$$

Requiring deformed solution to have the same asymptotics as (3.8) we have

$$
Z_{4}^{\infty}=0
$$

Small $\tau$ : we observe that

$$
\begin{aligned}
G^{a a}\left(\phi_{0}\right) e^{-4 p_{0}-4 A_{0}} & \sim \frac{1}{\tau^{2}}, \quad \text { for } \quad a=(1,2,3,4,6,8), \\
G^{77}\left(\phi_{0}\right) e^{-4 p_{0}-4 A_{0}} & =O(1), \quad G^{55}\left(\phi_{0}\right) e^{-4 p_{0}-4 A_{0}} \sim \tau^{2}, \\
h(\tau) & =h_{0}+h_{2} \tau^{2}+O\left(\tau^{4}\right),
\end{aligned}
$$

where $h_{0}$ and $h_{2}$ are constants. For $\xi(\tau)$ the leading asymptotics are

$$
\begin{aligned}
& \xi_{1}=\frac{1}{3} X_{\eta}^{0}-\frac{32}{27} \frac{P^{2} X_{\eta}^{0}}{h_{0}}+\frac{2}{3} \frac{X_{\eta}^{0} h_{2}}{h_{0}}-\frac{2}{5} P X_{7}^{0}+2 X_{1}^{0}+O\left(\tau^{2}\right), \\
& \xi_{2}=\frac{2}{3} \frac{X_{\eta}^{0} h_{2}}{h_{0}}-\frac{32}{27} \frac{P^{2} X_{\eta}^{0}}{h_{0}}-\frac{16}{15} P X_{7}^{0}+2 X_{1}^{0}+O\left(\tau^{2}\right), \quad \xi_{3}=-\frac{2}{3} X_{\eta}^{0}+O\left(\tau^{2}\right), \\
& \xi_{4}=-\frac{2}{3} X_{\eta}^{0}+\frac{32}{9} \frac{P^{2} X_{\eta}^{0}}{h_{0}}-2 \frac{X_{\eta}^{0} h_{2}}{h_{0}}+\frac{6}{5} P X_{7}^{0}-6 X_{1}^{0}+O\left(\tau^{2}\right), \\
& \xi_{5}=\frac{X_{7}^{0}}{2 \tau}+\frac{4 P X_{\eta}^{0}}{3 h_{0} \tau}+X_{5+6}^{0}+O(\tau), \quad \xi_{6}=-\frac{X_{7}^{0}}{2 \tau}-\frac{P X_{\eta}^{0} \tau}{9 h_{0}}+\frac{1}{12} X_{7}^{0} \tau+O\left(\tau^{2}\right), \\
& \xi_{8}=X_{8}^{0}+\frac{8 P_{7}^{2} X_{\eta}^{0} \tau^{2}}{27 h_{0}}+\frac{1}{6} X_{7}^{0}+O(\tau),
\end{aligned}
$$

where $X_{\eta}^{0}, X_{1}^{0}, X_{5+6}^{0}, X_{7}^{0}$ and $X_{8}^{0}$ are constants. The dilaton deformation is given by

$$
\bar{\Phi}=\frac{16}{\tau} X_{8}^{0}+Z_{8}^{0}+O(\tau)
$$

\footnotetext{
${ }^{1} \Gamma(a, z)=\int_{z}^{\infty} t^{a-1} e^{-t} d t$.
} 
where $Z_{8}^{0}$ is a constant. Regularity of $\bar{\Phi}$ at small $\tau$ implies $X_{8}^{0}=0$. We have

$$
\begin{aligned}
\bar{x}= & \frac{2}{\tau^{3}} Z_{2}^{0}+\frac{4}{3 \tau} X_{\eta}^{0}\left(1-\frac{20 P^{2}}{9 h_{0}}+\frac{2 h_{2}}{h_{0}}-\frac{8 P^{2} h_{2}}{3 h_{0}^{2}}+\frac{128 P^{4}}{27 h_{0}^{2}}\right)+\frac{8}{\tau} X_{1}^{0}\left(1-\frac{4 P^{2}}{3 h_{0}}\right)-\frac{4 P}{h_{0} \tau} Z_{5-6}^{0}+ \\
& +\frac{8 P}{\tau} X_{7}^{0}\left(-\frac{1}{5}+\frac{2 h_{2}}{h_{0}}+\frac{32 P^{2}}{45 h_{0}}\right)+\frac{88 P^{2}}{45 h_{0} \tau} Z_{2}^{0}-\frac{2 P}{h_{0} \tau} Z_{5+6}^{0}-Z_{3+4}^{0}+3 Z_{4}^{0}+O(\tau \log \tau), \\
\bar{y}= & -\frac{2}{\tau^{3}} Z_{2}^{0}+\frac{16}{3 \tau} X_{\eta}^{0}\left(\frac{h_{2}}{h_{0}}-\frac{16 P^{2}}{3 h_{0}}\right)+\frac{16}{\tau} X_{1}^{0}-\frac{128 P}{15 \tau} X_{7}^{0}+\frac{7}{3 \tau} Z_{2}^{0}+4 Z_{3+4}^{0}+O(\tau \log \tau), \\
\bar{p}= & \frac{1}{\tau^{3}} Z_{2}^{0}-\frac{4}{9 \tau} X_{\eta}^{0}\left(1+\frac{4 P^{2}}{3 h_{0}}-\frac{8 P^{2} h_{2}}{3 h_{0}^{2}}+\frac{128 P^{4}}{27 h_{0}^{2}}\right)+\frac{32 P^{2}}{9 h_{0} \tau} X_{1}^{0}-\frac{2}{15 \tau} Z_{2}^{0}\left(1+\frac{44 P^{2}}{9 h_{0}}\right)- \\
& -\frac{4 P^{2}}{3 \tau} X_{7}^{0}\left(1+\frac{4 h_{2}}{h_{0}}+\frac{64 P^{2}}{45 h_{0}}\right)+\frac{4 P}{3 h_{0} \tau} Z_{5-6}^{0}+\frac{2 P}{3 h_{0} \tau} Z_{5+6}^{0}+Z_{3+4}^{0}-Z_{4}^{0}+O(\tau \log \tau), \\
\bar{A}= & \frac{2}{15 \tau} Z_{2}^{0}\left(1+\frac{44 P^{2}}{9 h_{0}}\right)+\frac{4}{9 \tau} X_{\eta}^{0}\left(1-\frac{4 P^{2}}{h_{0}}+\frac{3 h_{2}}{h_{0}}-\frac{8 P^{2} h_{2}}{3 h_{0}^{2}}+\frac{128 P^{4}}{27 h_{0}^{2}}\right)-\frac{4 P}{3 h_{0} \tau} Z_{5-6}^{0}+ \\
& +\frac{4}{\tau} X_{1}^{0}\left(1-\frac{8 P^{2}}{9 h_{0}}\right)+\frac{P}{\tau} X_{7}^{0}\left(-\frac{4}{5}+\frac{16 h_{2}}{3 h_{0}}+\frac{256 P^{2}}{135 h_{0}}\right)-\frac{2 P}{3 h_{0} \tau} Z_{5+6}^{0}+Z_{4}^{0}+O(\tau), \\
\bar{f}= & \frac{1}{2} Z_{5-6}^{0}+\frac{1}{2} Z_{5+6}^{0}+O\left(\tau^{2}\right), \\
\bar{k}= & \frac{56 P}{5 \tau^{2}} Z_{2}^{0}+\frac{16 P}{3 \tau^{2}} X_{\eta}^{0}\left(1-\frac{2 h_{2}}{h_{0}}+\frac{32 P^{2}}{9 h_{0}}\right)- \\
& -\frac{32 P^{2}}{\tau^{2}} X_{1}^{0}+\frac{16}{\tau^{2}} X_{7}^{0}\left(3 h_{2}+\frac{16 P^{2}}{15}\right)-\frac{6}{\tau^{2}} Z_{5-6}^{0}-\frac{1}{2} Z_{5-6}^{0}+\frac{1}{2} Z_{5+6}^{0}+O(\tau \log \tau), \\
\bar{F}= & \frac{64 P}{15 \tau} Z_{2}^{0}+\frac{8 P}{3 \tau} X_{\eta}^{0}\left(1-\frac{2 h_{2}}{h_{0}}+\frac{32 P^{2}}{9 h_{0}}\right)-\frac{16 P}{\tau} X_{1}^{0}+\frac{2}{\tau} X_{7}^{0}\left(h_{0}+12 h_{2}+\frac{64 P^{2}}{15}\right)- \\
& -\frac{3}{\tau} Z_{5-6}^{0}+O(\tau),
\end{aligned}
$$

where $Z_{2}^{0}, Z_{3+4}^{0}, Z_{4}^{0}, Z_{5-6}^{0}$, and $Z_{5+6}^{0}$ are constants. The perturbation of the metric is small if

$$
2 \bar{p}-\bar{x}+2 \bar{A} \leq 0, \quad-6 \bar{p}-\bar{x} \leq 0, \quad \bar{x}+\bar{y} \leq 0, \quad \bar{x}-\bar{y} \leq 0 .
$$

Regularity of $\bar{f}, \bar{k}, \bar{F}$ together with (6.14) implies

$$
\begin{array}{rlrl}
Z_{2}^{0} & =X_{7}^{0}=0, & Z_{5-6}^{0}=\frac{8 P}{9} X_{\eta}^{0}, \\
X_{1}^{0}=\left(\frac{16 P^{2}}{27 h_{0}}-\frac{h_{2}}{3 h_{0}}\right) X_{\eta}^{0}, & Z_{5+6}^{0}=\left(\frac{2 h_{0}}{3 P}-\frac{8 P}{9}\right) X_{\eta}^{0} .
\end{array}
$$

The space of regular deformations: as far as we can see, it would require numerics on the coupled system (5.1) to describe any particular linearized deformation of the basic solution (3.8). However, having found the asymptotics both at large and small $\tau$, we can meaningfully inquire how many independent deformations there are, consistent with the basic ansatz, (1.1) and (3.2). There are fifteen integration constants for the system (2.1): seven $\{X\}$ and eight $\{Z\}$. At large $\tau$ we have constrained five of them: one of $\{X\}$ and four of $\{Z\}$, through dilaton asymptotics ([6.4), regularity conditions (6.6) and (5.9). 
At small $\tau$ regularity of $(6.12)$ and $(6.15)$ have constrained three of $\{X\}$ and three of $\{Z\}$. Two of these $\{Z\}$ are related to $\{X\}$ through (6.15). We have not shown that these are independent constraints, but on grounds of genericity we expect that this is so. According to our discussion in section 2 we conclude that there are three unconstrained real parameters, corresponding to three regular non-supersymmetric deformations.

This is not an easy result to understand directly from the field theory, partly because of the effects of strong coupling. The field content, in $\mathcal{N}=1$ superfield language, is

$\begin{array}{ccccc} & \mathrm{SU}(N) & \mathrm{SU}(N+M) & \mathrm{SU}(2)_{A} & \mathrm{SU}(2)_{B} \\ V_{1} & \operatorname{adj} & 1 & 1 & 1 \\ V_{2} & 1 & \operatorname{adj} & 1 & 1 \\ A & \bar{N} & N+M & 2 & 1 \\ B & N & \overline{N+M} & 1 & 2\end{array}$

where we have also indicated the quantum numbers under the gauge symmetry $\mathrm{SU}(N) \times$ $\mathrm{SU}(N+M)$ and the global symmetry $\mathrm{SU}(2)_{A} \times \mathrm{SU}(2)_{B}$. The first two lines show real superfields, while the second two show chiral superfields. Deformations of the supergravity solution of the type we consider should correspond to adding gauge theory operators to the lagrangian which are invariant under all the symmetries in (6.16). One difficulty with this point of view is that the theory is strongly coupled, in two ways: first, there is the usual large 't Hooft coupling limit associated with the geometry being smooth on the string scale; and second, the theory is close to a Leigh-Strassler fixed point with a quartic superpotential, so it's not clear that a simple lagrangian suffices to describe the physics. If we set this aside and write down a list of the gauge operators which are singlets under the symmetries in (6.16) and are relevant or marginal based on the dimensions one obtains from the Leigh-Strassler fixed point, there are considerably more than three: the list includes

$$
\begin{aligned}
& \operatorname{tr}\left[F_{\mu \nu}^{(1)}\right]^{2}, \quad \operatorname{tr}\left[F_{\mu \nu}^{(2)}\right]^{2}, \quad \operatorname{tr} \lambda_{(1)}^{2}, \quad \operatorname{tr} \lambda_{(2)}^{2}, \quad \operatorname{tr} \lambda_{(1)} \not D \lambda_{(1)}, \quad \operatorname{tr} \lambda_{(2)} \not D \lambda_{(2)}, \\
& \operatorname{tr}\left|a_{i}\right|^{2}, \quad \operatorname{tr}\left|b_{i}\right|^{2}, \quad \operatorname{tr}\left|D_{\mu} a_{i}\right|^{2}, \quad \operatorname{tr}\left|D_{\mu} b_{i}\right|^{2} \text {, } \\
& \operatorname{tr} \bar{\psi}_{a_{i}} \psi_{a_{i}}, \quad \operatorname{tr} \bar{\psi}_{b_{i}} \psi_{b_{i}}, \quad \operatorname{tr} \bar{\psi}_{a_{i}} \not D \psi_{a_{i}}, \quad \operatorname{tr} \bar{\psi}_{b_{i}} \not D \psi_{b_{i}}, \\
& \epsilon^{i k} \epsilon^{j l} \operatorname{tr} a_{i} b_{j} a_{k} b_{l}, \quad \epsilon^{i k} \epsilon^{j l} \operatorname{tr} \psi_{a_{i}} \psi_{b_{j}} a_{k} b_{l},
\end{aligned}
$$

where we have employed some obvious notation: $\lambda_{(1)}$ is the gaugino component of $V_{1}, a_{i}$ is the scalar component of $A_{i}, \psi_{a_{i}}$ is the fermion component of $A_{i}$, and the Roman indices $i, j, \ldots$ are labels for the fundamentals of $\mathrm{SU}(2)_{A}$ or $\mathrm{SU}(2)_{B}$. In writing down the list (6.17), we have incorporated also an R-symmetry constraint: $\mathrm{U}(1)_{R}$ is broken to a $\mathbb{Z}_{2}$ which should be respected by operators dual to the supergravity deformations that we have considered. Note that the fields $a_{i}$ and $b_{j}$ both have R-charge $1 / 2$ [6].

Two mechanisms cut down the list of operators that should appear in (6.17). First (and trivially), we constrained $\Phi(\infty)$, so only the difference $\operatorname{tr}\left[F_{\mu \nu}^{(1)}\right]^{2}-\operatorname{tr}\left[F_{\mu \nu}^{(2)}\right]^{2}$ should be allowed, not the sum. Second, some operators unrelated to chiral primaries must be expected to acquire large anomalous dimensions and to be dual to excited string states. It is not entirely clear even at the Leigh-Strassler fixed point which operators these are, but this is something that might be elucidated in the future. For a field theory with a moduli 
space of vacua, one might expect that a third mechanism would cut down the list of allowed deformations of the lagrangian, namely the requirement of a stable vacuum. However, as in the case of [14], the fact that the vacuum of the unperturbed theory is isolated tells us that any small deformation of the lagrangian should still have a stable vacuum. The upshot is that once one sorts out which operators correspond to supergravity modes as opposed to excited string states, it should be possible to identify the three allowed deformations on the gauge theory side. We hope to return to this problem in the future.

\section{Non-extremal deformation}

Because the gauge theory confines, we might reasonably expect that there are no nearextremal generalizations of the solution (3.8) with regular horizons. To understand the reasoning, let us first note that solutions with regular horizons do exist with the same asymptotics at infinity [27], but the horizon entropy of these solutions scales as $N^{2}$, and their gauge theory interpretation is in terms of a high temperature, deconfined phase with restored chiral symmetry. It shouldn't be possible for such a phase to persist arbitrarily close to zero energy density and temperature; hence the conclusion that near-extremal generalizations with regular horizons should not exist.

To explore this explicitly in our setup of linearized supergravity perturbations, let us make the following ansatz for the metric:

$$
\begin{aligned}
d s_{10}^{2}= & e^{2 p-x+2 A+2 z}\left(-e^{-8 z} d t^{2}+d x^{i} d x^{i}\right)+e^{2 p-x+8 A} d u^{2}+ \\
& +\left[e^{-6 p-x} g_{5}^{2}+e^{x+y}\left(g_{1}^{2}+g_{2}^{2}\right)+e^{x-y}\left(g_{3}^{2}+g_{4}^{2}\right)\right] .
\end{aligned}
$$

Let $\phi^{A}=\left(\phi^{a}, z\right)$ with $\phi^{a}=(x, y, p, A, f, k, F, \Phi)$. The reduced action has the form

$$
S\left[\phi^{A}\right]=-\frac{2 \mathrm{Vol}_{4}}{k_{5}^{2}} \int d u\left(-\frac{1}{2} G_{A B} \phi^{A} \phi^{B}-V(\phi)\right),
$$

where

$$
G_{A B} \phi^{\prime A} \phi^{\prime B}=6 z^{\prime 2}+G_{a b} \phi^{\prime a} \phi^{\prime b},
$$

and the potential $V(\phi)$, the superpotential $W(\phi)$, and the metric $G_{a b}$ are the same as in section 月:

$$
V=\frac{1}{8} G^{A B} \partial_{A} W \partial_{B} W=\frac{1}{8} G^{a b} \partial_{a} W \partial_{b} W .
$$

The constraint equation has the following form:

$$
G_{a b}\left(\phi^{\prime a}-\frac{1}{2} G^{a c} \partial_{c} W\right)\left(\phi^{\prime b}+\frac{1}{2} G^{b d} \partial_{d} W\right)+6=0 .
$$

The supersymmetric solution satisfies

$$
\phi^{\prime A}=\frac{1}{2} G^{A B} \partial_{B} W .
$$

We notice that both the target-space metric $G_{A B}$ and superpotential $W$ are independent of $z$, which implies that $z$ is constant in the supersymmetric solution and can be absorbed into redefinition of $t$ and $x^{i}$. 
Let

$$
\phi^{A}=\phi_{0}^{A}+\alpha \hat{\phi}^{A}+\alpha^{2} \tilde{\phi}^{A}+O\left(\alpha^{3}\right)
$$

where $\alpha$ is a deformation parameter and $z_{0}=0$. Equation $\delta S / \delta z=0$ implies $z^{\prime \prime}=0$. To study the non-extremal deformations we choose

$$
z=\alpha \bar{z}, \quad \bar{z}=u .
$$

Substitution of (7.7) into the equations of motion and constraint gives (to first order in $\alpha$ )

$$
\frac{d}{d u} g_{a}+g_{b} N_{a}^{b}\left(\phi_{0}\right)=0, \quad g_{a} \phi_{0}^{\prime a}=0
$$

where

$$
g_{a}=G_{a b}\left(\phi_{0}\right)\left(\hat{\phi}^{b}-N_{d}^{b}\left(\phi_{0}\right) \hat{\phi}^{d}\right), \quad N_{a}^{b}=\frac{1}{2} \partial_{a}\left(G^{b c} \partial_{c} W\right) .
$$

Equations (7.9) and (7.10) do not involve $z$ and correspond to the extremal deformation of the supersymmetric solution. To study non-extremal deformation we set $\hat{\phi}^{a}=0$. For $\tilde{\phi}^{a}$ we have

$$
\frac{d}{d u} \zeta_{a}+\zeta_{b} N_{a}^{b}\left(\phi_{0}\right)=0, \quad \zeta_{a} \phi_{0}^{\prime a}+3=0
$$

where

$$
\zeta_{a}=G_{a b}\left(\phi_{0}\right)\left(\tilde{\phi}^{\prime b}-N_{d}^{b}\left(\phi_{0}\right) \tilde{\phi}^{d}\right) .
$$

Solutions for $\zeta$ and $\tilde{\phi}$ can be written in terms of the general solution for $\xi$ and $\bar{\phi}$. For small $\tau$ we have

$$
\begin{array}{rlrl}
\zeta_{1} & =-\frac{12}{\tau}-4 \tau+\xi_{1}+O\left(\tau^{2}\right), & \zeta_{2} & =-\frac{6}{\tau}-\frac{43}{5} \tau+\xi_{2}+O\left(\tau^{2}\right), \\
\zeta_{3} & =\frac{12}{\tau}-\frac{28}{5} \tau+\xi_{3}+O\left(\tau^{2}\right), & \zeta_{4} & =\frac{48}{\tau}+\frac{32}{5} \tau+\xi_{4}+O\left(\tau^{2}\right), \\
\zeta_{5} & =\xi_{5}+O(\tau), & \zeta_{6} & =\xi_{6}+O\left(\tau^{2}\right), \\
\zeta_{7} & =\xi_{7}+O(\tau), & \zeta_{8} & =\xi_{8}+O\left(\tau^{3}\right), \\
\tilde{x} & =-\frac{16}{\tau^{2}}+64 \log \tau-\frac{424}{15}+\bar{x}+O(\tau \log \tau), & & \\
\tilde{y} & =-\frac{16}{\tau^{2}}+128 \log \tau-\frac{824}{15}+\bar{y}+O(\tau \log \tau), & \\
\tilde{p} & =\frac{8}{3 \tau^{2}}-\frac{128 P^{2}}{3 h_{0}} \log \tau+\frac{256 P^{2}}{27 h_{0}}+\bar{p}+O(\tau \log \tau), & \\
\tilde{A} & =-\frac{32}{3 \tau^{2}}+\left(32+\frac{128 P^{2}}{3 h_{0}}\right) \log \tau-\frac{256 P^{2}}{27 h_{0}}+\bar{A}+O(\tau), & \\
\tilde{f}=32 P \tau+\bar{f}+O\left(\tau^{2}\right), & \tilde{k}=-\frac{64 P}{3 \tau}+\bar{k}+O(\tau \log \tau), \\
\tilde{F} & =-\frac{16}{3} P+\bar{F}+O(\tau), & \tilde{\Phi}=\bar{\Phi}+O(\tau) .
\end{array}
$$

We observe that for any values of the integration constants, a function $\tilde{k}$ is infinite at $\tau=0$. Therefore, we conclude that non-extremal linearized deformations (7.7) are singular at the apex of deformed conifold. Thus we reach the desired conclusion that near-extremal solutions with regular horizons do not exist. 


\section{Acknowledgments}

V.B. would like to thank Arkadas Ozakin, Xinkai Wu, and Takuya Okuda for useful discussions. The work of V.B. was supported in part by the DOE under grant DE-FG0392ER40701. The work of S.S.G. was supported in part by the DOE under grants DEFG03-92ER40701 and DE-FG02-91ER40671 and through an Outstanding Junior Investigator Award. S.S.G. gratefully acknowledges Caltech's support while this work was carried out.

\section{References}

[1] J.M. Maldacena, The large- $N$ limit of superconformal field theories and supergravity, Adv. Theor. Math. Phys. 2 (1998) 231 hep-th/9711200.

[2] S.S. Gubser, I.R. Klebanov and A.M. Polyakov, Gauge theory correlators from non-critical string theory, Phys. Lett. B 428 (1998) 105 hep-th/9802109.

[3] E. Witten, Anti-de Sitter space and holography, Adv. Theor. Math. Phys. 2 (1998) 253 hep-th/9802150.

[4] O. Aharony, S.S. Gubser, J.M. Maldacena, H. Ooguri and Y. Oz, Large-N field theories, string theory and gravity, Phys. Rept. 323 (2000) 183 hep-th/9905111.

[5] E. Witten, Anti-de Sitter space, thermal phase transition and confinement in gauge theories, Adv. Theor. Math. Phys. 2 (1998) 505 hep-th/9803131.

[6] I.R. Klebanov and M.J. Strassler, Supergravity and a confining gauge theory: duality cascades and $\chi S B$-resolution of naked singularities, J. High Energy Phys. 08 (2000) 052 hep-th/0007191.

[7] J.M. Maldacena and C. Núñez, Towards the large- $N$ limit of pure $N=1$ super Yang-Mills, Phys. Rev. Lett. 86 (2001) 588 hep-th/0008001.

[8] S.S. Gubser, Dilaton-driven confinement, hep-th/9902155.

[9] L. Girardello, M. Petrini, M. Porrati and A. Zaffaroni, The supergravity dual of $N=1$ super Yang-Mills theory, Nucl. Phys. B 569 (2000) 451 hep-th/9909047.

[10] S.S. Gubser, Curvature singularities: the good, the bad and the naked, Adv. Theor. Math. Phys. 4 (2002) 679 hep-th/0002160.

[11] J.M. Maldacena and C. Núñez, Supergravity description of field theories on curved manifolds and a no go theorem, Int. J. Mod. Phys. A 16 (2001) 822 hep-th/0007018.

[12] M. Grana and J. Polchinski, Supersymmetric three-form flux perturbations on AdS $S_{5}$, Phys. Rev. D 63 (2001) 026001 hep-th/0009211.

[13] S.S. Gubser, Supersymmetry and F-theory realization of the deformed conifold with three-form flux, hep-th/0010010.

[14] O. Aharony, E. Schreiber and J. Sonnenschein, Stable non-supersymmetric supergravity solutions from deformations of the maldacena-Núñez background, J. High Energy Phys. 04 (2002) 011 hep-th/0201224.

[15] S.S. Gubser, A.A. Tseytlin and M.S. Volkov, Non-abelian 4-d black holes, wrapped 5-branes and their dual descriptions, J. High Energy Phys. 09 (2001) 017 hep-th/0108205. 
[16] N. Evans, M. Petrini and A. Zaffaroni, The gravity dual of softly broken $N=1$ super Yang-Mills, J. High Energy Phys. 06 (2002) 004 hep-th/0203203.

[17] S. Ferrara, R. Kallosh and A. Strominger, $N=2$ extremal black holes, Phys. Rev. D 52 (1995) 5412 hep-th/9508072.

[18] D.Z. Freedman, S.S. Gubser, K. Pilch and N.P. Warner, Renormalization group flows from holography supersymmetry and a c-theorem, Adv. Theor. Math. Phys. 3 (1999) 363 hep-th/9904017.

[19] M. Wijnholt and S. Zhukov, On the uniqueness of black hole attractors, hep-th/9912002.

[20] O. DeWolfe, D.Z. Freedman, S.S. Gubser and A. Karch, Modeling the fifth dimension with scalars and gravity, Phys. Rev. D 62 (2000) 046008 hep-th/9909134.

[21] K. Skenderis and P.K. Townsend, Gravitational stability and renormalization-group flow, Phys. Lett. B 468 (1999) 46 hep-th/9909070.

[22] J. de Boer, E. Verlinde and H. Verlinde, On the holographic renormalization group, J. High Energy Phys. 08 (2000) 003 hep-th/9912012.

[23] L.A. Pando Zayas and A.A. Tseytlin, 3-branes on resolved conifold, J. High Energy Phys. 11 (2000) 028 hep-th/0010088.

[24] V. Balasubramanian and P. Kraus, A stress tensor for anti-de Sitter gravity, Commun. Math. Phys. 208 (1999) 413 hep-th/9902121.

[25] M. Bianchi, D.Z. Freedman and K. Skenderis, How to go with an RG flow, J. High Energy Phys. 08 (2001) 041 hep-th/0105276.

[26] M. Bianchi, D.Z. Freedman and K. Skenderis, Holographic renormalization, Nucl. Phys. B 631 (2002) 159 hep-th/0112119.

[27] S.S. Gubser, C.P. Herzog, I.R. Klebanov and A.A. Tseytlin, Restoration of chiral symmetry: a supergravity perspective, J. High Energy Phys. 05 (2001) 028 hep-th/0102172. 